Matthias Hüning

\title{
Wortbildung im niederländisch-deutschen Sprachvergleich
}

\section{Zusammenfassung}

Im vorliegenden Beitrag werden einige zentrale Aspekte der kontrastiven Wortbildungsforschung anhand von Beispielen aus dem niederländisch-deutschen Sprachvergleich besprochen. Als nah verwandte Sprachen zeigen das Niederländische und das Deutsche zwar vergleichbare Strukturen der Komposition und der Derivation, bei näherem Hinsehen sind es aber vor allem die vielfältigen Divergenzen, die ins Auge fallen. Im ersten Teil des Artikels werden verschiedenartige Beispiele für solche Divergenzen besprochen. Anschließend geht es um gegenläufige Entwicklungen, die zu Konvergenz zwischen beiden Sprachen führen. Anhand einer Analyse von Zusammensetzungen vom Typ wassergekühlt (ndl. watergekoeld) wird für eine stärkere Berücksichtigung von Konvergenzfaktoren in der kontrastiven Linguistik plädiert. Der zweite Teil des Artikels enthält eine detaillierte Fallstudie zur Adjektivbildung mit dem niederländischen Suffix -achtig und dem deutschen - haft, die den Zusammenhang von diachronen Entwicklungen und synchronen Kontrasten aufzeigt. Zudem zeigt sie Konsequenzen und Implikationen der vergleichenden Analyse für die Theorie des Lexikons und der Wortbildung auf. Im Mittelpunkt stehen dabei Rainers (2003) Idee der ,semantischen Fragmentierung' von Wortbildungsmustern und die Konzeption eines, hierarchischen Lexikons', wie sie unter anderem von Jackendoff (2008) oder Booij (2010) vertreten wird.

\section{Einleitung ${ }^{1}$}

Das Deutsche und das Niederländische kennzeichnen sich im Bereich der Wortbildung durch eine große Übereinstimmung im Hinblick auf die verwendeten Verfahren und Muster. Durch die enge genetische Verwandtschaft haben sich weitgehend vergleichbare Strukturen der Komposition und der Derivation herausgebildet. Dennoch finden sich bei genauerer Betrachtung vielfältige Divergenzen in der Verwendung dieser Möglichkeiten.

Eine umfassende Studie zur vergleichenden Betrachtung der Wortbildung des Niederländischen und des Deutschen ist mir nicht bekannt. Die vorhandenen Arbeiten beschränken sich auf einen Ausschnitt (wie beispielsweise der vergleichende Überblick von Hüning/Schlücker 2010 zur Komposition) oder aber auf den Vergleich einzelner Wortbildungsmuster (wie Tellenbach 1976 zum Präfix ver-). Einen hervorragenden (englischsprachigen) Überblick über die Morphologie des Niederländischen bietet Booij (2002).

Ich danke den Teilnehmern der IDS-Jahrestagung für die stimulierende Diskussion meines Vortrags und Geert Booij für seinen Kommentar zu einer früheren Version dieses Artikels. Wertvolle Hinweise verdanke ich auch den beiden Gutachtern für diesen Band. 
Im vorliegenden Beitrag sollen einige Aspekte der kontrastiven Wortbildungsforschung anhand von Beispielen und kleineren Fallstudien zum deutsch-niederländischen Vergleich besprochen werden. Der Artikel verfolgt ein zweifaches Ziel: Zum einen soll er zeigen, dass es für ein angemessenes Verständnis von Übereinstimmungen und Kontrasten zwischen verwandten Sprachen wünschenswert (oder auch notwendig) ist, die historische Dimension einzubeziehen. Zum anderen geht es mir darum zu zeigen, dass die kontrastive Analyse von Wortbildungsmustern auch in theoretischer Hinsicht einen relevanten Beitrag leisten kann.

Eine zentrale Frage jeder sprachvergleichenden Untersuchung ist die nach dem ,tertium comparationis‘. Man muss sich fragen, was die Basis des Vergleichs sein soll, um - ausgehend von dieser Grundlage - zu Aussagen über Unterschiede und Übereinstimmungen gelangen zu können. Im Bereich der Wortbildung gibt es grundsätzlich zwei verschiedene Möglichkeiten, ein ,tertium comparationis' zu bestimmen und damit eine Vergleichsgrundlage zu schaffen, die allerdings in der Praxis in aller Regel miteinander kombiniert werden.

Im Bereich der derivationellen Morphologie liegt es - zumindest für verwandte Sprachen - nahe, einander formal bzw. etymologisch entsprechende Affixe als Ausgangspunkt zu nehmen und die Möglichkeiten der Wortbildung mit diesen Affixen in den untersuchten Sprachen miteinander zu vergleichen. Dieser semasiologischen Betrachtungsweise steht die onomasiologische gegenüber, bei der wir eine bestimmte Bedeutung oder eine bestimmte Funktion zugrunde legen und uns ansehen, ob und wie diese in den untersuchten Sprachen realisiert wird.

In seinem Buch „Contrasting Languages: The Scope of Contrastive Linguistics" plädiert Krzeszowski für diese zweite Möglichkeit:

Since formal comparisons of individual lexical items do not seem to lend themselves to any significant generalizations, contrastive studies of word formation are better off if they are based on some conceptual framework. [...] As a matter of fact, any aspect of the meaning can serve as a basis for cross-linguistic comparisons. (Krzeszowski 1990, S. 75)

Prinzipiell scheint es richtig und sinnvoll, in sprachvergleichenden Untersuchungen von der Bedeutung auszugehen und die formalen Wortbildungsmöglichkeiten zum Ausdruck dieser Bedeutung zu inventarisieren und miteinander zu vergleichen. Dennoch sollte man meines Erachtens auch die Möglichkeiten des semasiologischen Vergleichs nicht unterschätzen. Gerade bei eng verwandten Sprachen wie Niederländisch und Deutsch lassen sich einzelne Wortbildungsmuster oft direkt zueinander in Beziehung setzen. Sie können historisch miteinander identifiziert werden, und es ist aufschlussreich zu beobachten, ob und wie sie sich in beiden Sprachen parallel oder aber unterschiedlich entwickelt haben. Der historische Blickwinkel ermög- 
licht es vielfach, synchrone Übereinstimmungen und Parallelen bzw. synchrone Unterschiede diachron als Resultat konvergierender bzw. divergierender Entwicklungen zu analysieren und zu erklären.

\section{Konvergenz und Divergenz von Wortbildungsmustern}

Direkte Äquivalenzen zwischen Affixen und Wortbildungsmustern gibt es beim Vergleich des Niederländischen mit dem Deutschen beispielsweise bei:

(1) deverbalen Nomina agentis mit -er (de spreker, de bakker - der Sprecher, der Bäcker)

(2) deverbalen Nomina actionis und Abstrakta mit -ing bzw. -ung (de handeling, de ervaring - die Handlung, die Erfabrung)

(3) Adjektiven mit -baar bzw. -bar auf der Basis transitiver Verben (afwasbaar, leesbaar-abwaschbar, lesbar)

Hier korrespondieren strukturell vergleichbare formale Möglichkeiten mit identischer oder doch zumindest sehr vergleichbarer Semantik. Selbst bei diesen strukturellen Äquivalenzen gibt es im Detail jedoch viele kleine Unterschiede und Besonderheiten. (vgl. beispielsweise den Umlaut bei Bäcker oder die Allomorphie zwischen -er und -aar im Niederländischen: loochenen ,leugnen' - loochenaar ,Leugner). Die in den genannten Beispielen unterstellte Äquivalenz der strukturellen Möglichkeiten führt nie zur vollständigen Äquivalenz auf lexikalischem Niveau. Und ganz allgemein kann man wohl festhalten, dass es - wenn überhaupt - dann nur im Ausnahmefall eine strukturelle Isomorphie, ein wirkliches Eins-zu-eins-Verhältnis, zwischen Sprachen gibt.

Das andere Extrem ist das Fehlen einer bestimmten Wortbildungsmöglichkeit in einer der beiden Sprachen. Auch solche synchronen Kontraste können bei nah verwandten Sprachen oft aus der Diachronie heraus als Divergenzen beschrieben und unter Umständen auch erklärt werden.

\subsection{Divergenz}

Vergleicht man das System der verbalen Wortbildung im Niederländischen mit dem Deutschen, so fällt auf, dass dem Niederländischen eine Entsprechung für das deutsche Verbalpräfix zer-fehlt.

(4) Deutsch [zer- + V $]_{\mathrm{V}}$ zerbeißen, zerbrechen, zerbröckeln, zerkochen, zermablen, zerscblagen, zerschneiden, zerstechen, zerteilen etc. 
Die Übersetzungsäquivalente für derartige Verben zeigen, dass es im Niederländischen nicht ein äquivalentes Wortbildungsverfahren gibt; es werden verschiedene sprachliche Mittel gewählt um den entsprechenden Inhalt zu kodieren.

(5) Beispiele verbaler zer-Bildungen mit niederländischen Entsprechungen eine Nuss zerbeißen - een noot stukbijten/doorbijten die Mauern zerbersten - de muren barsten das Eis zerfließt - het ijs smelt (weg) die Farben zerfließen - de kleuren vervloeien

Der Blick in die Sprachgeschichte zeigt aber, dass das direkte Äquivalent zu zer-im Mittelniederländischen durchaus vorhanden war und auch produktiv genutzt wurde. Das Mittelniederländische hatte ein Verbalpräfix te-, das dem deutschen zer- sowohl im Hinblick auf die Bildungsweisen als auch auf die Funktion der präfigierten Verben genau entsprach:

(6) Mittelniederländisch [te- $+\mathrm{V}]_{\mathrm{V}}$

tebersten, zerbersten', tebiten ,zerbeißen', tebreken ,zerbeißen', teblouwen ,zerschlagen', tegaen ,zergehen', teslaen ,zerschlagen', testeken ,zerstechen', testoren, zerstören' etc.

Eine genaue historische Analyse macht deutlich, welche Faktoren zum Verlust der Produktivität des Verfahrens und zum Verschwinden des Präfixes geführt haben können, und wie die Funktionen dieses Wortbildungsmusters dann mit anderen sprachlichen Mitteln ausgedrückt werden. Die Erklärung für das Verschwinden des niederländischen Präfix muss meines Erachtens vor allem in der Form gesucht werden (vgl. hierfür die ausführlichere Analyse in Hüning 1997). Im Deutschen verschmolz das ursprüngliche Präfix za-/ze-mit dem Präfix ar-/er- (man denke hier an die Verwendung eines Wortes wie erbrechen im Sinne von aufbrechen: ,einen Brief/ein Schloss erbrechen ${ }^{2}{ }^{2}$ Dadurch wurde es lautlich vom Infinitivmarkierer $z u$ unterschieden (noch im Althochdeutschen ebenfalls $; a / z e)$. Im Niederländischen dagegen behielten beide Element, also das Präfix wie auch der Infinitivmarkierer, die Form te. Dadurch konnte es zu einem direkten Aufeinandertreffen der beiden Formen kommen:

(7) Infinitiv: te breken (,zu brechen'), Präfix: tebreken (,zerbrechen'), Infinitiv + Präfix: te tebreken (,zu zerbrechen')

Da die Verwendung der Infinitivmarkierung mit te (das auf die Präposition beim Verbalabstraktum zurückgeht) im Laufe des Mittelniederländischen immer stärker obligatorisch wurde, konnte die lautliche Entsprechung durch-

$2 \quad$ Vgl.: „₹a- setzte sich vor ar-, das häufig eine dem $z^{a}$ - nahe stehende bedeutung entwickelt hatte" (Grimm 1854-1960, Bd. 31, S. 646). 
aus zu einem hemmenden Faktor für die Verwendung des Präfixes werden. Der ,Horror Aequi‘ (Plank 1981) führte zur Vermeidung des Präfixes im Niederländischen bzw. zur Ersetzung durch andere sprachliche Mittel und schließlich zum Verlust dieser Wortbildungsmöglichkeit. Im Deutschen, wo die Verwendung des $z$-Infinitivs ebenfalls stark zunahm, entstand aufgrund der Differenzierung in $z u$ und zer- keine Vermeidungstendenz und das Präfix blieb bis in die heutige Zeit erhalten. ${ }^{3}$

Eine solche Form der Divergenz stellt aber im deutsch-niederländischen Sprachvergleich eher die Ausnahme dar. Weitaus häufiger findet man den Fall, dass morphologische Verfahren zwar strukturell in beiden Sprachen vorhanden sind, aber unterschiedlich genutzt werden bzw. wurden. ${ }^{4}$

So zeigt sich bei einer semasiologischen Untersuchung der deverbalen Nomina auf -sel, dass es in beiden Sprachen Äquivalenzen gibt (,das, was überbleibt ${ }^{6}$ ist im Deutschen ein Überbleibsel und im niederländischen ein overblijfsel), daneben aber auch die Verwendung konkurrierender oder komplementärer Bildungsweisen. So können mit dem niederländischen -sel Wörter mit resultativer bzw. passivischer Bedeutung gebildet werden, die im Deutschen meist eine Entsprechung mit -ung haben (wie die Anschwemmung oder Beimischung). ${ }^{5}$ Auch andere Entsprechungen sind möglich, wie die Beispiele unter (b) zeigen.

$[\mathrm{V}+\mathrm{sel}]_{\mathrm{N}}$

(a) -selim Niederländischen und im Deutschen:

het aanhangsel - das Anhängsel

het overblijfsel-das Überbleibsel

(b) -sel nur im Niederländischen:

het aanslibsel - die Anschwemmung

het afdruksel-der Abdruck

het bedenksel-die (reine) Erfindung

het bezinksel-der Bodensatz

het bijmengsel-die Beimischung

het smeersel-die Schmiere

3 Dass Vermeidungstendenzen wie die hier beschriebene nicht absolut aufzufassen sind, zeigen die deutschen Partikelverben mit zu-, bei denen sich in Verbletztstellung ebenfalls zu-zuFolgen ergeben können (,Er beabsichtigt, bei diesem Verfahren nur zu₹usehen ${ }^{2}$ ). Eine genauere vergleichende Untersuchung müsste zeigen, ob sich Faktoren identifizieren lassen, die im Fall des niederländischen Präfixes zur Vermeidung geführt haben, im Fall der deutschen Partikel aber nicht. (Mit Dank an den/die Gutachter/in, der/die mich auf diese zu-zu-Folgen im Deutschen hingewiesen hat.)

4 Ein klassisches Beispiel sind die A+N-Komposita, die in beiden Sprachen möglich sind (Dünndruck - dundruk), aber im Niederländischen weniger verwendet werden. Dem deutschen Kompositum entspricht dann die syntaktische Phrase (Rotwein - rode wijn). Vgl. hierzu u.a. Hüning (2010) und die dort zitierte Literatur.

$5 \quad$ Vgl. aber die regionale Verwendung des passivischen Füllsel für Füllung (,das, was (in eine Speise) eingefüllt wird'). 
(c) -sel nur im Deutschen:

das Mitbringsel - het cadeautje

das Einsprengsel - hier en daar voorkomend deeltje

Die Beispiele unter (c) machen deutlich, dass es andererseits auch -sel-Ableitungen im Deutschen gibt, die keine direkte Entsprechung im Niederländischen haben (*medebrengsel oder *insprengsel sind keine Wörter des Niederländischen).

Wählt man statt der semasiologischen eine onomasiologische Herangehensweise, dann trifft man ebenfalls häufig auf ein recht heterogenes Bild. Die Korrespondenz zwischen Funktionen/Bedeutungen und Formen ist keineswegs immer in der gleichen oder in gleichartiger Weise gegeben. Als Beispiel möge hier die Movierung dienen. Schaut man sich die Bildung weiblicher Personenbezeichnungen im Deutschen und im Niederländischen an, so zeigt sich, dass dem sehr uniformen und regelmäßigen -in im Deutschen eine Vielzahl von Bildungsmöglichkeiten im Niederländischen entspricht.

(9) Movierung im Deutschen und im Niederländischen

(a) Held/Held $i n$ - held/heldin Bauer/Bäurin - boer/boerin

(b) Schwimmer/Schwimmerin - zwemmer/zwemster Spaziergänger/-gängerin - wandelaar/wandelaarster

(c) Lehrer/Lehrerin - leraar/lerares Sänger/Sängerin - zanger/zangeres

(d) Dozent/Dozentin - docent/docente Fotograf/Fotografin - fotograaf/fotografe

(e) Masseur/Masseurin - masseur/massense Akteur/Akteurin - acteur/actrice

(f) Arzt/Ärztin - arts/vrouwelijke arts Minister/Ministerin - minister/vrouwelijke minister Ingenieur/Ingenieurin - ingenieur/vrouwelijke ingenieur

Das Suffix -in wird im Niederländischen nur für eine Handvoll Wörter verwendet (vgl. (a)); es ist nicht mehr produktiv. Es hat sich eine etwas unübersichtliche Vielfalt von Wortbildungsmöglichkeiten mit unterschiedlichen Suffixen entwickelt (produktiv sind insbesondere -ster und -e). Zudem gibt es neben additiven Mustern, bei denen das Suffix an die männliche Form der Personenbezeichnung angehängt wird, auch Affixsubstitution, wie in masseur - masseuse oder acteur - actrice. Bei diesen Fällen paradigmatischer Wortbildung wird das männliche Suffix durch das weibliche ersetzt. ${ }^{6}$ Die ent-

Auch das Suffix -ster wird in paradigmatischer Weise zur Ersetzung von -er verwendet. Es wird nicht an das männliche -er angehängt (wohl aber an das Allomorph -aar, wie wandelaarster zeigt), sondern ersetzt dies in Wörtern wie spemster. Der paradigmatische Charakter der Movierung mit -ster zeigt sich insbesondere bei nicht-deverbalen Personenbezeichnungen wie vrijwilliger/vrijwilligster (das Adjektiv vrijwillig bedeutet ,freiwillig', ein korrespondierendes Verb gibt es nicht). 
sprechenden deutschen Formen (Masseuse, Dompteuse, Friseuse usw.) werden zunehmend durch das additive Muster mit -in ersetzt (Masseurin, Dompteurin, Friseurin). Und schließlich ist im Niederländischen für einige Personenbezeichnungen die Bildung der weiblichen Form mit morphologischen Mitteln schlicht unmöglich (arts, minister, professor). Die verschiedenen Bildungsmöglichkeiten stehen in Konkurrenz zueinander, die Wahl des jeweiligen Suffixes wird u.a. durch das Suffix des Basiswortes bestimmt.

Während die Movierung im Deutschen im Laufe der Zeit stark vereinheitlicht worden ist, kennzeichnet sich das Niederländische durch ,formale Fragmentierung' für die Bildung weiblicher Personenbezeichnungen: Dieselbe Funktion wird durch unterschiedliche morphologische Mittel realisiert (oder eben auch nicht, wie bei den Beispielen in (f)). Die systematische Kennzeichnung und Verwendung der weiblichen Form im Deutschen ist zum einen Resultat gesellschaftlicher Entwicklungen in der zweiten Hälfte des 20. Jahrhunderts. Daneben spielt aber sicher auch eine Rolle, dass im Deutschen das Geschlecht auch bei der Wahl des Artikels kodiert werden muss und zudem Konsequenzen für die Flexion hat. Da im Niederländischen Maskulinum und Femininum zusammengefallen sind (im Utrum), spielt die Unterscheidung eine weniger wichtige Rolle. Damit ist das Genus ein Beispiel für die allgemeine Einsicht, dass „languages differ essentially in what they must convey, not in what they may convey ". ${ }^{8}$ Prinzipiell kann man alles in allen Sprachen sagen, aber Sprachen unterscheiden sich erheblich im Hinblick darauf, welche Unterscheidungen kodiert werden müssen. Im Deutschen gehört das Geschlecht dazu: Es ist gegenwärtig zumindest sehr markiert, wenn man vom ,Bundeskanzler Merkel' spricht, während ,kanselier Merkel' auf Niederländisch oder, Chancellor Merkel' im Englischen (wo der Zusammenfall der Genera noch weiter fortgeschritten ist) völlig neutral verwendet werden können, ohne dass das Geschlecht kodiert werden müsste.

Divergenz ist bei sprachvergleichenden Untersuchungen zur Wortbildung eher Normalfall als Ausnahme: Sowohl die semasiologische Perspektive (Beispiel -sel) als auch die onomasiologische (Movierung) zeigt vielfältige Unterschiede in der Ausgestaltung des Wortbildungssystems, auch bei nah verwandten Sprachen. Während die diachrone Analyse dazu beitragen kann, Faktoren und Sprachwandelprozesse zu identifizieren, die den heutigen $\mathrm{Zu}$ stand herbeigeführt haben, stellt sich für die synchrone kontrastive Analyse die Herausforderung, zu sinnvollen Generalisierungen zu kommen, auch beispielsweise im Hinblick auf den Fremdsprachenerwerb.

Vgl. für eine ausführliche Analyse der Möglichkeiten im Niederländischen Booij (2002), insbesondere Paragraph 3.2.3.

8 Guy Deutscher in einem Interview mit „The Paris Review“ über sein neues Buch (Deutscher 2010), vgl. www.theparisreview.org/blog/2010/11/09/ (Stand: 20.05.2011). Ich danke Geert Booij für den Hinweis auf dieses Interview. 
In Abschnitt 3 werde ich noch etwas ausführlicher auf ein weiteres Beispiel von Divergenz im Bereich der Adjektivbildung eingehen und dabei die synchrone Perspektive mit der diachronen kombinieren, um zu Einsichten über das Funktionieren von Wortbildung und Lexikon zu gelangen.

Zunächst möchte ich jetzt aber darauf hinweisen, dass es durchaus auch gegenläufige Tendenzen in der Wortbildung gibt. Es ist keineswegs so, dass das Wortbildungssystem aufgrund von Sprachwandelprozessen im Laufe der Zeit immer heterogener oder chaotischer wird. Es gibt zahlreiche Entwicklungen, die eher parallel laufen und zu Konvergenz führen.

\subsection{Konvergenz}

Konvergenz zwischen Sprachen findet im Bereich des Lexikons und der Wortbildung unter anderem durch Sprachkontakt und Entlehnungen statt. Fanden im Laufe der Geschichte vor allem Entlehnungen aus dem Lateinischen und dem Französischen Eingang in die anderen europäischen Sprachen, so ist es momentan vor allem das Englische, das als Quelle für viele ,Europäismen' und ,Internationalismen' dient. Es geht dabei um Wörter, die international bekannt sind und in die jeweiligen Sprachen übernommen werden. Mit den Wörtern werden auch Affixe entlehnt und diese können in den aufnehmenden Sprachen als solche erkannt und genutzt werden.

Auffällig ist, dass das Lateinische und das Griechische immer noch sehr populär sind als ,Lieferant ‘ für neue internationale Affixe. Neue Wörter mit Präfixen und Präfixoiden wie den folgenden illustrieren das (hier in niederländischer Schreibweise):

$$
\begin{aligned}
& \text { ante-, anti-, astro-, auto-, bi-, biblio-, bio-, contra-, cosmo-, eco-, ex-, extra-, fysio-, } \\
& \text { bydro-, hyper-, hypo-, inter-, macro-, maxi-, micro-, mini-, mono-, multi-, neo-, } \\
& \text { non-, onmi-, paleo-, pan-, para-, pluri-, poly-, post-, pre-, pro-, pseudo-, psycho-, } \\
& \text { radio-, sub-, super-, tele-, thermo-, trans-, ultra-, uni-, video-, ... }
\end{aligned}
$$

Dass diese Elemente in vielen europäischen Sprachen produktiv verwendet werden, kann als Konvergenzfaktor gesehen werden. Es findet eine Art von Ausgleich durch internationalen Kontakt statt. Menschen verwenden diese Elemente in ihren jeweiligen Sprachen, insbesondere für fachsprachliche Terminologie und Fachjargon. Es entsteht eine Art europäischer bzw. internationaler Wortschatz (vgl. Wandruszka 1998, S. 9). Diese Entwicklung betrifft natürlich auch das Niederländische und das Deutsche und führt zu Konvergenz im Bereich des Wortschatzes.

Daneben gibt es aber auch Konvergenz ohne direkte Entlehnung von konkreten Wörtern. Als Beispiel möchte ich Zusammensetzungen vom Typ wassergekühlt, computergesteuert anführen, Zusammensetzungen mit der Struktur $[\mathrm{N}+\text { Partizip II }]_{\mathrm{A}}$ also. ${ }^{9}$

Die folgenden Ausführungen basieren auf Hüning/Schlücker (2010), wo dieser Wortbildungstyp (in Par. 3.2) ausführlicher besprochen und vergleichend analysiert wird. 
Die semantischen Möglichkeiten dieses Zusammensetzungsmusters sind im Deutschen wie im Niederländischen ausgesprochen vielfältig. Sie ergeben sich aus dem semantischen Verhältnis des nominalen Erstglieds zum verbalen zweiten Glied des Kompositums (Fleischer/Barz 1992; PümpelMader et al. 1992; van den Toorn 1984).

(11) instrumental: watergekoelde motor/wassergekühlter Motor , mit Wasser gekühlt

final: toekomstgericht beleid/ zukunftsgerichtete Politik , auf die Zukunft gerichtet

lokativ: buisgemaakte soep/ hausgemachte Suppe ,im Haus gemacht ${ }^{6}$ agentiv: maanverlicht/mondbeschienen, vom Mond beschienen“ kausal-agentiv: milieubepaald gedrag/ umweltbestimmtes Verbalten, durch die Umwelt bestimmt ${ }^{6}$ ornativ: sneeuwbedekte bergen/schneebedeckte Berge, mit Schnee bedeckt ${ }^{6}$ konsekutiv-graduativ: hoogglansgepolijst/hochglan₹poliert ,auf/zu Hochglanz poliert ${ }^{6}$

limitativ-relational: geluidgedempte compressor/schallgedämpfter Kompressor , in Bezug auf den Schall gedämpt ${ }^{6}$

material: houtgesneden pop/ holiggeschnitzte Puppe , aus Holz geschnitzt ${ }^{6}$

In beiden Sprachen haben wir es also gegenwärtig mit einem produktiven und semantisch vielfältigen Wortbildungsmuster zu tun. Für das Niederländische ist wiederholt darauf hingewiesen worden, dass dieses Wortbildungsmuster vor allem in den vergangenen Jahrzehnten produktiv verwendet worden ist. Van der Horst/van der Horst (1999, S. 339) stellen fest, dass das Niederländische in den letzten Jahrzehnten des 20. Jahrhunderts „,von einer Lawine solcher Zusammensetzungen überschüttet" worden sei, und van den Toorn (1984) konstatiert ebenfalls eine deutliche Zunahme von Zusammensetzungen dieses Typs. Auch für das Deutsche wird behauptet, dass der Typ vor allem in den vergangenen Jahrzehnten produktiv verwendet worden ist. Wilss (1986, S. 168) ist gar der Meinung, dass diese Zusammensetzungen erst in der zweiten Hälfte des 20. Jahrhunderts zu einem eigenständigen Wortbildungsmuster der deutschen Sprache geworden sind.

Dennoch ist man sich in der Literatur dahingehend einig, dass es sich um eine grundsätzlich sehr alte germanische Wortbildungsmöglichkeit handelt. Der Typ ist im Althochdeutschen und vor allem im Altenglischen gut belegt, kam dann aber offensichtlich ein wenig aus der Mode: Im Mittelhochdeutschen, Mittelenglischen und Mittelniederländischen finden sich nur wenige Beispiele. Erst gegen Ende des Mittelalters findet man wieder erste Neubildungen (Carr 1939; Koziol 1972; van den Toorn 1984). Der Wortbildungstyp wurde langsam, wiederbelebt', und vor allem im Laufe des 20. Jahrhunderts gewann er immer mehr an Popularität (vgl. auch Marchand 1969, S. 92). 
Die Wiederbelebung dieses Wortbildungsmusters scheint im Englischen, Deutschen und Niederländischen parallel zu verlaufen. ${ }^{10}$ Zwar ist für das Niederländische wiederholt auf einen möglichen Einfluss durch Entlehnungen aus dem Deutschen und/oder dem Englischen hingewiesen worden (u.a. von van der Sijs 1996, S. 299-300), aber, Entlehnung' scheint mir für die Erklärung solcher Parallelen nicht hinreichend und auch nicht zutreffend zu sein.

Sicherlich sind einzelne Wörter zwischen den Sprachen entlehnt worden (z.B. Luthers schriftgelehrt bzw. die Substantivierung der Schriftgelehrte in das Niederländische: de schriftgeleerde), es kann aber wohl keine Rede davon sein, dass der Wortbildungstyp als solcher im 20. Jahrhundert entlehnt wurde. Allenfalls haben einzelne Entlehnungen dazu beigetragen, einem vorhandenen, aber kaum oder nicht produktiven Wortbildungsmuster wieder zu Popularität und Produktivität zu verhelfen. Statt von Entlehnung ist daher wohl eher von einer parallel verlaufenden Entwicklung auszugehen. Es scheinen sich in den verschiedenen Sprachen parallele Ausdrucksbedürfnisse entwickelt zu haben, die zur vermehrten Verwendung dieses Kompositionstyps geführt haben. Die offenbar stark zunehmende Produktivität des Wortbildungsmusters in den germanischen Sprachen im Laufe des 19. und vor allem des 20. Jahrhunderts weist auf neue Ausdrucksnotwendigkeiten hin. Viele der Neubildungen sind fachsprachlich zuzuordnen. Häufig geht es zwar um durchaus gängige Wörter, die aber dennoch kaum als umgangssprachlich bezeichnet werden können: die witterungsangepasste Fahrweise, der schülerzentrierte Unterricht, der wassergekühlte Motor, die rechnergestützte Auswertung - es sind fachsprachliche Ausdrücke, die im „Prozeß der Verwissenschaftlichung unserer Erfahrung" über die Medien auch in die Gemeinsprache übernommen werden (vgl. hierzu die Ausführungen bei Wilss 1986, Kapitel IX zu Sprache und Umwelt). Für das Niederländische weist die Algemene Nederlandse Spraakkunst darauf hin, dass Komposita von Substantiv + Partizip II ,vor allem in der Sprache von Technik und Handel vorkommen", wie z.B. geluidgedempt, schallgedämpft', handgeweven , handgewebt' oder luchtgekoeld ,luftgekühlt' (Haeseryn et al. 1997, S. 729).

Da die Komposita im Vergleich zu korrespondierenden syntaktischen Konstruktionen kurz und prägnant sind und zudem oft einen plakativen Aspekt haben, finden sie sich auch vermehrt in der Sprache der Werbung: die calgon-gepflegte Waschmaschine, die TÜV-geprïfte Internetsicherbeit usw. Für das Niederländische finden sich viele Beispiele bei van den Toorn (1984, S. 405), wie die Citroën-Werbung für de nieune schijfgeremde eend, die neue scheibengebremste Ente' oder für gasgevulde schokdempers, gasgefüllte Stoßdämpfer‘.

10 Zusammensetzungen vom Typ computergesteuert finden sich nicht nur im Deutschen, Niederländischen (computergestuurd) oder Englischen (computer-controlled), sondern auch in den skandinavischen Sprachen (z.B. Dänisch computerstyret, Norwegisch komputerstyrt). 
Derartige kommunikative Bedürfnisse sind ganz offensichtlich heutzutage nicht (mehr) einzelsprachlich zuzuordnen, sie erfassen in Zeiten der Internationalisierung und Globalisierung zeitgleich verschiedene Sprachen und Sprachgebiete. Zunehmender Kontakt und die gemeinsame Kontaktsprache Englisch sorgen dabei für konvergierende Entwicklungen in den Sprachen. Die Sprecher bauen parallel gleiche oder vergleichbare Strategien aus, um die gesellschaftlichen Umstände und Neuerungen sprachlich angemessen verarbeiten zu können.

Der Begriff der ,Entlehnung wird diesen Entwicklungen kaum gerecht. Es geht um die Konvergenz sprachlicher Entwicklungen, die durch den direkten Sprachvergleich augenfällig wird, häufig aber - wie auch in diesem Fall - nicht nur zwei Sprachen betrifft, sondern international relevant ist. Ein brauchbares Konzept für die Beschreibung solcher Entwicklungen könnte vielleicht das der, replication' sein, wie es von Heine und Kuteva in ihrem Buch „The changing languages of Europe“ vorgeschlagen wurde. Darunter verstehen sie, dass sich in einer Sprache eine grammatische/lexikalische Struktur herausbildet, für die eine andere Sprache Modell steht. „It concerns meanings and the structures associated with them, but not forms, that is, phonetic substance is not involved" (Heine/Kuteva 2006, S. 49). Dadurch unterscheidet sich die Replikation von der Entlehnung, bei der sehr wohl auch phonetische Substanz übernommen wird. Sie weisen aber selber darauf hin, dass Entlehnung und Replikation nicht komplett unterschiedliche Phänomene sind: „Still, borrowing and replication are not always independent of one another [...] it may also happen that borrowing provides a basis for replication" (ebd., S. 72). Die Entstehung bzw. der Ausbau des besprochenen Wortbildungstyps kann meines Erachtens als Beispiel einer derartigen ,Replikation' analysiert werden.

Eine genauere Untersuchung könnte Aufschluss über die Datierung und die relative Reihenfolge der einzelsprachlichen Entwicklungen geben. Sie würde zudem zeigen können, dass und warum der Produktivitätsschub die verschiedenen Sprachen fast gleichzeitig erfasst hat. Man müsste sich fragen, in welchen Bereichen des Wortschatzes die Zunahme der Produktivität sich ereignet hat. Ist es möglich, plausible Motive und Gründe für die Popularität des Wortbildungsmusters zu identifizieren? Hat es einzelsprachliche Sonderentwicklungen gegeben (und wie sind diese gegebenenfalls zu erklären)? Erst die genaue Untersuchung derartiger Fragen ermöglicht meines Erachtens ein vertieftes Verständnis von Konvergenz und Divergenz in der Wortbildung und von Wortbildungswandel allgemein.

Die kontrastive Sprachwissenschaft richtet ihr Augenmerk oft fast ausschließlich auf die Kontraste und die Divergenzen. Es wäre wünschenswert, dass wir auch in vergleichenden Untersuchungen zur Wortbildung etwas mehr Aufmerksamkeit für die Konvergenzen und die Parallelen entwickeln. 
Die Typologie ist mit ihren Untersuchungen zum ,Standard Average European' der kontrastiven Sprachwissenschaft hier weit voraus (vgl. unter anderem König/Haspelmath 1999; Haspelmath 2001; Heine/Kuteva 2006).

Nach diesen Überlegungen zu konvergierenden Entwicklungen in der Wortbildung komme ich jetzt zu einer Fallstudie zur Adjektivbildung im Deutschen und im Niederländischen, bei der wieder eher die Divergenz zwischen beiden Sprachen im Mittelpunkt steht. Konkret geht es um die etymologisch verwandten Suffixe dt. -haft und ndl. -achtig. Ziel dieser Fallstudie ist es, noch einmal etwas detaillierter auf den Zusammenhang von diachronen Entwicklungen und synchronen Kontrasten einzugehen. Zudem soll auf die Konsequenzen und Implikationen der vergleichenden Analyse für die Theorie des Lexikons und der Wortbildung eingegangen werden. Insbesondere unterstützt die Fallstudie meines Erachtens die Idee eines hierarchischen Lexikons, wie sie unter anderem von Jackendoff (2008) und Booij (2010) vertreten wird.

\section{Fallstudie: Adjektive mit dt. -haft/ndl. -achtig}

Im Bereich der Adjektivbildung gibt es diverse Verfahren und Muster, die einander teils komplementär ergänzen, teils überlappen und zueinander in Konkurrenz stehen. Im Sprachvergleich ergibt sich daraus eine hochkomplexe, kaum überschaubare Variation von möglichen Entsprechungen zwischen den diversen Wortbildungsmustern. Ausgehend vom niederländischen Suffix -achtig sollen im Folgenden einige dieser Entsprechungen aufgezeigt werden. Anschließend werde ich die theoretischen Implikationen der vorgelegten Analyse erläutern.

Das niederländische Adjektivsuffix -achtig ist ein polyfunktionales Affix: Es kann mit Basiswörtern verschiedener Wortarten kombiniert werden. Zudem ergeben sich - abhängig von der Wortart und der Bedeutung der Basis - verschiedene semantische Interpretationen. Booij (2002, S. 105) unterscheidet die folgenden Möglichkeiten:

$$
\begin{array}{ll}
\text { A-achtig } & \text {,somewhat } \\
\text { N-achtig } & \text {,like' } \\
& \text {,liking' } \\
& \text {,having a lot } \\
\text { V-achtig } & \text {,inclined to }
\end{array}
$$

groen-achtig ,greenish ${ }^{6}$ aap-achtig , like a monkey ${ }^{6}$ pasta-achtig , liking pasta ${ }^{\circ}$ rots-achtig, rocky ${ }^{6}$ weiger-achtig , unwilling'

Etymologisch ist -achtig mit dem deutschen Adjektivsuffix -haft verwandt (Maesfranckx/Taeldeman 1998; Pijnenburg 1993). Die unterschiedlichen Frikative erklären sich aus einem regelhaften Lautwandel im Altniederländischen (ab dem 10. Jahrhundert), den man auch anderswo findet:

$$
\begin{aligned}
& \text { /ft/ } \rightarrow \text { / xt/ } \\
& \text { dt. Luft, stiften, heften - ndl. lucht, stichten, hechten }
\end{aligned}
$$


Zudem fällt im Hinblick auf die Form auf, dass das niederländische Suffix schon früh (ebenfalls im Altniederländischen) mit dem Suffix -ig kombiniert worden ist. Offenbar konnte der adjektivische Charakter der Ableitungen durch die pleonastische Kombination mit -ig noch verdeutlicht werden, ein Fall von ,Übercharakterisierung ${ }^{6}$ also. ${ }^{11}$ Von der entsprechenden Form gibt es auch im Deutschen noch einige Reste (leibhaftig, wahrhaftig). Zudem weist auch die Nominalisierung der -haft-Adjektive darauf hin, dass die Erweiterung mit -ig in den älteren Stufen des Deutschen wohl sehr viel prominenter war als heute. Adjektive mit -haft werden nämlich nicht mit -keit sondern mit -igkeit nominalisiert (also nicht nur Wabrhaftigkeit, sondern auch Dauerhaftigkeit, Lückenhaftigkeit, Regelhaftigkeit usw., bei denen die Erweiterung mit -ig beim Adjektiv nicht (mehr) vorkommt). ${ }^{12}$

Der Verlust des anlautenden /h/ erklärt sich aus dem Zusammenfall des alten -baft mit dem Suffix -echt (vgl. noch dt. töricht). Beide Suffixe waren laut Pijnenburg (1993) schon im 13. Jahrhundert weitestgehend zusammengefallen. Ich komme auf diesen Zusammenfall der beiden Suffixe noch zurück, da er für den Vergleich mit dem deutschen -baft von zentraler Bedeutung ist.

Nicht nur das niederländische -achtig, auch das deutsche -baft kann mit Wörtern verschiedener Wortarten kombiniert werden, um neue Adjektive zu bilden. Bei den denominalen Ableitungen findet sich häufig das Fugenelement -en (wie in professorenhaft), wenn das entsprechende Basissubstantiv dieses Morphem zur Pluralbildung verwendet. In der Regel kann es auch synchron als Pluralmorphem interpretiert werden. Daneben finden sich auch einige Ableitungen mit dem Fugenelement $-s$ (früblingshaft).

\subsection{Denominale Vergleichsbildungen}

Für die denominalen -haft-Adjektive nennt Eichinger als zentrale Funktion die Vergleichsbildung und er weist dabei auch auf die Konkurrenz mit -ig hin: Die -haft-Ableitungen ,setzen ein deutlicheres Signal für Eigenschaftsbezeichnungen aus einem Vergleich, als das die funktional durchaus vergleichbaren Entsprechungen mit -ig tun" (Eichinger 2000, S. 213). In dieser Bedeutung entsprechen sie den niederländischen -achtig-Bildungen:

(14) Denominale Vergleichsbildungen

ein bünenhafter Kerl - een reusachtige kerel

schurkenhaftes Verhalten - schurkachtig gedrag

eine albtraumbafte Erzählung - een nachtmerrieachtig verhaal

schlangenhafte Bewegungen - slangachtige bewegingen

\footnotetext{
11 Ich übernehme den Begriff overkarakterisering (,Übercharakterisierung) hier von Van Marle (1978, S. 148).

12 Vgl. Fleischer/Barz (1992, S. 158 ff.) zur Distribution von -heit, -keit und -igkeit.
} 
ein früblingshafter Tag - een lenteacbtige dag eine romanhafte Biographie - een romanacbtige biografie ein orgelhafter Klang - een orgelachtige klank die panikhafte Reaktion - de paniekachtige reactie

Ein bünenbafter Kerl ist ein Kerl wie ein Hüne, schurkacbtig gedrag ist Verhalten, das dem Verhalten eines Schurken ähnelt, schlangenhafte Bewegungen ähneln den Bewegungen einer Schlange und eine romanachtige biografie ist eine Biographie, die Züge eines Romans trägt.

Untersucht man die beiden Wortbildungsmuster nun aber im Hinblick auf ihre Erweiterbarkeit, auf ihre Produktivität, so zeigt sich, dass die Möglichkeiten der Vergleichsbildungen im Niederländischen größer sind als im Deutschen. Als Input für Ableitungen mit -achtig kommen Substantive verschiedenster Art in Betracht, wobei die deutsche Entsprechung mit -haft in vielen Fällen eher zweifelhaft oder aber gar unmöglich erscheint. So sind die folgenden Neubildungen im Niederländischen durchaus möglich, ${ }^{13} \mathrm{im}$ Deutschen aber wohl nicht:

$$
\begin{aligned}
& \text { een tafelpootachtig voorwerp - ??ein tischbeinhaftes Objekt } \\
& \text { een terrasachtig balkon - ?? ein terrassenhafter Balkon } \\
& \text { olieachtige vloeistof - ?? ?eine ölhafte Flüssigkeit } \\
& \text { een Google-achtige zoekmachine - ??eine Google-hafte Suchmaschine } \\
& \text { een melkachtige smaak - ??ein milchhafter Geschmack }
\end{aligned}
$$

Für die Neubildung derartiger Vergleichsbildungen kommt im Deutschen eher -artig in Betracht als -baft: ein tischbeinartiges Objekt, ein terrassenartiger Balkon, eine Google-artige Suchmaschine oder ein milchartiges Getränk klingen sehr viel überzeugender als die Ableitungen mit -baft. Alternativ sind auch $\mathrm{Zu}-$ sammensetzungen mit beispielsweise -ähnlich möglich: terrassenähnlicher Balkon usw. Und ein melkachtige smaak wäre wohl ein milchartiger oder aber ein milchiger Geschmack.

Die Produktivität des denominalen Musters mit -baft bzw. -achtig ist also nicht in gleicher Weise gegeben. Die Möglichkeiten der Ausbreitung sind im Deutschen restringierter als im Niederländischen.

Wenn man aber die Klasse der Basisnomina weiter eingrenzt, dann scheint es doch eine Äquivalenz zwischen den beiden Wortbildungsmustern zu geben, einen Bereich, in dem die Muster in beiden Sprachen produktiv verwendet werden können. Handelt es sich nämlich bei dem Basissubstantiv um eine Personenbezeichnung, dann ist im Deutschen die Vergleichsbildung mit -haft genauso produktiv möglich wie im Niederländischen mit -achtig.

13 Die hier und im Folgenden verwendeten Beispiele sind alle belegt, entweder in Korpora oder über Google, wobei darauf geachtet wurde, dass dann jeweils mehrere Belege aus unterschiedlichen Quellen vorhanden sind. 
(16) divenhaft - diva-achtig

eselhaft-ezelachtig

flegelhaft - vlegelachtig

gaunerhaft - schurkachtig

knabenhaft - jongensachtig

lausbubenhaft - kwajongensachtig

mädchenhaft - meisjesachtig

professorenhaft - professorachtig

riesenhaft - reusachtig

schülerhaft - schooljongensachtig/schoolmeisjesachtig

zwerghaft - dwergachtig

etc.

Und auch für Neubildungen wie freakhaft/freakachtig oder nerdhaft/nerdachtig lassen sich bei Google leicht viele Beispiele finden.

freakhaftes Verhalten - freakachtig gedrag

das kommt etwas nerdhaft rüber - dat komt een beetje nerdachtig over

In diesem semantisch enger gefassten Rahmen finden wir also tatsächlich die systematische Entsprechung der beiden Wortbildungsmuster.

Auffällig ist, dass genau für diese Gruppe von Basiswörtern die ansonsten häufige synonymische Konkurrenz mit -artig nur relativ selten angetroffen wird. Die Bemerkung von Fleischer/Barz (1992, S. 234), dass die Konkurrenz zwischen - haft und -artig bei Vergleichsbildungen mit Personenbezeichnungen als Basis nicht vorkommt, ist zwar so nicht zutreffend, doch finden sich selbst bei Google nur wenige Belege für Wörter wie professorenartig. Und den über 100.000 Google-Treffern für divenhaft stehen gerade mal ca. 500 für divenartig gegenüber. Bei Neubildungen wie freakhaft/freakartig ist das Verhältnis zwar etwas ausgeglichener, aber auch hier überwiegt - haft sehr deutlich. ${ }^{14}$

Wir haben es hier also mit einer semantisch kohärenten Untergruppe der denominalen Vergleichsbildungen zu tun, die in beiden Sprachen produktiv erweiterbar ist und in der sich die beiden Suffixe -baft und -achtig systematisch entsprechen.

\subsection{Deadjektivische Ableitungen}

Wir haben gesehen, dass -achtig (anders als - haft) auch für einen weiteren Typ der Vergleichsbildungen produktiv verwendet werden kann, und zwar für deadjektivische Ableitungen. Für das deutsche - haft finden sich nur sehr vereinzelt Adjektive als Basis (wie boshaft, krankhaft oder wahrhaft). Fleischer/

14 Bei denominalen - haft-Ableitungen, deren Basiswort keine Personenbezeichnung ist, ist das Verhältnis meist umgekehrt: Neben einer Ableitung wie panikhaft kommt auch - und zwar sehr viel häufiger - panikartig vor. Ausnahmen bilden lexikalisierte Formen wie früblingshaft. 
Barz (1992, S. 256) weisen darauf hin, dass in diesen Fällen zudem historisch Substantive zugrunde liegen (mhd. bōs ,Bosheit', kranc ,Krankheit' und wàr, Wahrheit'). Das niederländische -acbtig dagegen ist auch auf der Basis von Adjektiven durchaus produktiv:

$$
\begin{aligned}
& \text { Deadjektivische Ableitungen mit -achtig } \\
& \text { bitterachtig - leicht bitter } \\
& \text { donkerachtig - etwas dunkel } \\
& \text { doofachtig - ein wenig taub } \\
& \text { mageracbtig - etwas mager } \\
& \text { ziekachtig - kränklich } \\
& \text { zoetachtig - süßlich } \\
& \text { zunrachtig - säuerlich }
\end{aligned}
$$

Viele dieser niederländischen Ableitungen sind nicht sehr gebräuchlich und konkurrieren mit anders gebildeten Synonymen (wie zoetig oder ziekelijk). Andere, wie bitteracbtig, sind durchaus gängig. Sie haben eine abschwächende, relativierende Bedeutung. Ein bitterachtige drank ist ein Getränk mit leicht bitterem Geschmack. Deutsche Entsprechungen verwenden, wenn es sie denn gibt, in der Regel das Suffix -lich (zoetachtig, zuurachtig - süßlich, säuerlich), ansonsten kann die Relativierung der Bedeutung des Basisadjektivs syntaktisch realisiert werden, durch Hinzufügung eines relativierenden Elements (wie leicht, ein wenig, etwas usw.).

Auffällig ist, dass es auch innerhalb des deadjektivischen Musters eine semantisch kohärente Untergruppe gibt, bei der die Korrespondenz sehr regelmäßig ist, nämlich die Farbadjektive. Hier gibt es eine direkte Entsprechung: nicht zwischen -achtig und -baft, sondern zwischen -acbtig und -lich:

$$
\begin{aligned}
& \text { Farbadjektive } \\
& \text { blawwachtig - bläulich } \\
& \text { bruinachtig - bräunlich } \\
& \text { geelachtig - gelblich } \\
& \text { grijsachtig - gräulich } \\
& \text { groenachtig - grünlich } \\
& \text { roodacbtig - rötlich } \\
& \text { witacbtig - weißlich } \\
& \text { zwartacbtig - schwär₹lich }
\end{aligned}
$$

Allerdings ist die Entsprechung nicht ganz hundertprozentig, im Niederländischen sind die Möglichkeiten etwas größer. Auch Bezeichnungen für Mischfarben können mit -acbtig relativiert/abgeschwächt werden, wovon abgeleitete Adjektive wie paarsacbtig, violettfarben' oder lila-achtig , lilafarben' zeugen. ${ }^{15}$ Insgesamt lässt sich aber festhalten, dass sich auch bei den deadjek-

15 Ein/e Gutachter/in schlägt vor, dass dies damit zu tun haben könnte, dass -lich ein ,echtes Suffix ist, wohingegen -acbtig in formaler Hinsicht eher einem Suffixoid ähnelt (zweisilbig, vol- 
tivischen Adjektiven eine semantische Nische herausgebildet hat, in der in beiden Sprachen recht konsistent ein bestimmtes Affix verwendet wird und für die sich im Sprachvergleich die systematische Korrespondenz zwischen zwei Wortbildungsmustern (-achtig und -lich) feststellen lässt.

\subsection{Denominale Ornative}

Kehren wir zurück zu den denominalen Ableitungen. Die zweite Hauptbedeutung, die für die Muster mit -baft und -achtig angetroffen wird, lässt sich wohl am ehesten als, ornativ' umschreiben. Man kann sie paraphrasieren als

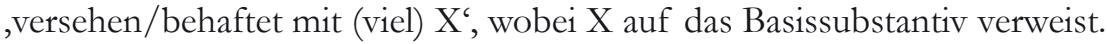
Booij hatte als Paraphrase angegeben, having a lot of ' (vgl. (12)).

Fleischer/Barz (1992, S. 256) weisen darauf hin, dass diese ,ornativen Ableitungen mit -haft ausschließlich Nicht-Personenbezeichnungen als Basis haben. Als Beispiele führen sie Wörter wie skrupelhaft (,mit Skrupeln behaftet') an. Der Vergleich einzelner Ableitungen dieses Typs mit den jeweiligen Übersetzungsäquivalenten im Niederländischen zeigt ein recht diffuses Bild:

$$
\begin{aligned}
& \text { Ornative Ableitungen } \\
& \text { fehlerhaft - foutief } \\
& \text { fieberhaft - koortsachtig } \\
& \text { krampfhaft - krampachtig } \\
& \text { schambaft - beschaamd } \\
& \text { schwunghaft - levendig, swingend } \\
& \text { skrupelhaft - scrupuleus } \\
& \text { tugendhaft - deugdzaam } \\
& \text { vorteilhaft - voordelig }
\end{aligned}
$$

Die wenigen Beispiele machen deutlich, dass hier offensichtlich kaum von einer eindeutigen Entsprechung zwischen -haft und -achtig gesprochen werden kann. Entsprechungen gibt es zwar auf Wortniveau, auf dem kategorialen Niveau kann man aber nicht von einer systematischen Entsprechung zwischen den beiden Suffixen für die Bildung ornativer Ableitungen sprechen.

Dreht man die Perspektive um und geht vom Niederländischen aus, dann gibt es aber innerhalb des niederländischen denominalen Musters der -achtig-Ableitungen mit ornativer Bedeutung wieder eine Gruppe von semantisch eng zusammengehörigen Wörtern, die im Deutschen sehr wohl eine systematische Entsprechung kennen, allerdings nicht mit -haft.

Es geht um Adjektive, die man als ,topographische Adjektive' bezeichnen könnte, und deren Entsprechungen im Deutschen alle mit -ig gebildet sind.

ler Vokal). Das in lautlicher Hinsicht vergleichbare -artig scheint bei derartigen Basiswörtern tatsächlich eher möglich als die Ableitung mit -lich (*lilalich, *violettlich vs. lilaartig, violettartig). 
(21) Topographische Adjektive

Niederländisch: heuvelachtig, bergachtig, steenachtig, struikachtig, rotsachtig, waterachtig

Deutsch: bügelig, bergig, steinig, strauchig, felsig, wässrig

Diese Adjektive können verwendet werden, um eine Landschaft oder eine Gegend zu charakterisieren, die sich durch die Anwesenheit des durch das Grundwort Bezeichneten auszeichnet.

Wiederum gibt es also eine semantisch kohärente Untergruppe, für die sich eine systematische Entsprechung zwischen dem Niederländischen und dem Deutschen feststellen lässt, diesmal zwischen -acbtig und -ig. Diese Korrespondenz lässt sich historisch erklären, und zwar durch den weiter oben schon angesprochenen Zusammenfall zweier Suffix im niederländischen -achtig.

\subsection{Der doppelte Ursprung des niederländischen -achtig}

Das Suffix -haft entspricht dem gotischen Partizip hafts (zu hafjan, heben'). Bis ins Alt- und Mittelhochdeutsche findet man haft in der Bedeutung, gefesselt, gebunden' (vgl. etwa Gotisch lingom hafts, durch Ehe gebunden, verheiratet') (Henzen 1965, S. 207-208; Wilmanns 1899, S. 501). Paul (1920, S. 100) gibt als ursprüngliche Bedeutung von -haft an , mit etwas behaftet, versehen'. Er weist aber darauf hin, dass „,der Sinn von -baft früh verblaßt, so daß man es etwa durch -artig ersetzen könnte, und so werden denn Wörter gebildet, die zu der Grundbedeutung von -baft nicht mehr passen, vgl. fabel-, mädchen-, mann-, märchen- meister-, [...]“ Die ursprüngliche Bedeutung findet sich in den heutigen Ornativen wie tugendhaft, zweifelhaft, ekelhaft, fehlerhaft usw. Henzen (1965, S. 208) merkt an, dass die Adjektivableitungen mit - haft heute in ihren Möglichkeiten ,beschränkt' sind; produktiv sind seiner Auffassung nach vor allem noch die Ableitungen, aus persönlichen Substantiven' (also die genannte Gruppe vom Typ professorenhaft).

Für die Geschichte des niederländischen -achtig ist nun neben diesem -baft ein weiteres Suffix wichig, das es im Deutschen ebenfalls gab. Ursprünglich hatte es die Form -abs, die man auch im Gotischen noch findet (bairgahs, bergig', steinabs, steinig'). Das Suffix wurde mit -t erweitert und hatte im Althochdeutschen die Form -ocht (boumocht, baumreich). Im Mittelhochdeutschen wurde diese Form meist zu -echt abgeschwächt.

(22) Mittelhochdeutsche Ableitungen mit -ocbt/-echt

bartoht ,bärtig', bërgeht ,bergig', bûcheht ,bauchig', buschobt ,buschig', velseht ,felsig', glatzeht ,glatzköpfig', knorrobt ,knorrig', lockeht ,lockig', moseht , mosig' usw. 
Wie die neuhochdeutschen Übersetzungen der Beispiele schon deutlich machen, wurde -ocht/-echt im Laufe der weiteren Sprachgeschichte recht systematisch durch das Suffix -ig ersetzt, das mit der entsprechenden Bedeutung auch im Alt- und Mittelhochdeutschen schon gängig war. Das alte Suffix wurde im 17. und 18. Jahrhundert noch produktiv verwendet (vgl. Ableitungen wie blumicht, schatticht, runzlicht, zotticht usw.); heute findet man es nur noch in töricht (von Tor ,Narr) (vgl. zur Geschichte dieses Suffixes Wilmanns 1899, S. 467-468; Paul 1920, S. 97; Henzen 1965, S. 199-200).

Beide Suffixe, -haft und -echt, gab es auch im Altniederländischen. Durch den oben angesprochenen altniederländischen Lautwandel wurde -haft zu -hacht. Aufgrund der semantischen Nähe (beide Suffixe wurden zur Bildung von Adjektiven mit ornativer Bedeutung verwendet), fielen -hacht und -echt dann in der Form -acht zusammen (Pijnenburg 1993). Dieses Suffix wurde (semantisch redundant) um das Suffix -ig erweitert, das ebenfalls ornativ verwendet werden konnte. Diese Erweiterung findet sich übrigens auch bei den beiden ursprünglichen Suffixen (also -echtich und -haftig). Wie oben schon erwähnt, betraf diese ,Übercharakterisierung' auch das Deutsche: -haft konnte in älteren Sprachstufen zu -haftig erweitert werden, und im Mittelhochdeutschen finden sich neben den -ebt-Ableitungen auch „pleonastische Weiterbildungen auf -ig wie knorrebtic, narrebtic, tôrebtic" (Paul 1920, S. 97).

Pijnenburg (1993, S. 46) fasst die Geschichte des niederländischen Suffix -achtig so zusammen:

Der doppelte Ursprung des niederländischen -achtig

(a) - echtig $<$-echt + -ich (Typ bergachtig)

(b) -(h)achtig < -haftig < -haft + -ig (Typ waarachtig)

Der Zusammenfall von (a) und (b) war im 13. Jahrhundert weitgehend vollzogen.

Die Geschichte des niederländischen -achtig und seines deutschen Pendants -haft zeigt, dass partielle Entsprechungen häufig historisch motiviert sind. Der doppelte Ursprung von -acbtig zeigt sich bis heute insbesondere in der Entsprechung bei den ,topographischen Adjektiven', die im Niederländischen mit -acbtig gebildet werden, im Deutschen aber mit -ig (das an die Stelle des alten -ocht/-echt getreten war).

Derartige Entwicklungen in den beiden untersuchten Sprachen führen zu Divergenz, das Resultat ist auf den ersten Blick oft sehr unübersichtlich: Es scheint keine oder kaum systematische Entsprechungen auf Wortbildungsniveau zu geben. Hinzu kommt, dass die Geschichte der Adjektivableitungen schon sehr früh von vielfältigen Konkurrenzen zwischen verschiedenen Wortbildungsmustern gekennzeichnet ist, die im Laufe der Geschichte teilweise zusammenfallen (Henzen 1965, S. 208). Hierbei sind aber, wie wir gesehen haben, deutliche sprachspezifische Unterschiede zu beobachten. Zu- 
sammenfall von Suffixen und semantische Fragmentierung führen dazu, dass die beteiligten Wortbildungsmuster sich heute nur noch in Teilabschnitten entsprechen, bei formal und insbesondere semantisch bestimmbaren Gruppen von Basiswörtern. Auch hier ist die Entsprechung auf dem lexikalischen Niveau oft nicht 1:1, aber es lassen sich ,Inseln' bestimmen, in denen sich zwei Muster in den beiden Sprachen entsprechen. Das haben wir oben beispielsweise für die denominalen Adjektive von Personenbezeichnungen festgestellt (mit -haft und -achtig), für die ,topographischen Adjektive' (mit -ig und -achtig) und auch für die Farbadjektive (mit -lich und -achtig). Und teilweise, wie im Fall der Ableitungen von Personenbezeichnungen mit -haft, können diese Inseln auch als ,Produktivitätsinseln' aufgefasst werden: Sie definieren einen Bereich, in dem das Muster produktiv verwendet werden kann und dem ebenfalls produktiven Muster der anderen Sprache entspricht.

\subsection{Weitere Typen}

Zum Abschluss dieses Vergleichs der Adjektivbildung mit -haft und -achtig möchte ich noch kurz auf die deverbalen Ableitungen hinweisen. Auch hier ist die Situation im Hinblick auf die Entsprechungen zwischen dem Deutschen und dem Niederländischen unübersichtlich. Für beide Sprachen gilt zudem, dass der deverbale Typ nicht mehr produktiv verwendet wird.

$$
\begin{aligned}
& \text { Deverbale Ableitungen mit -baft } \\
& \text { flatterhaft - wispelturig } \\
& \text { glaubhaft - geloofwaardig } \\
& \text { naschhaft - snoepachtig, }{ }^{16} \text { snoepziek } \\
& \text { schmeichelhaft - vleiend, geflatteerd } \\
& \text { schmerzhaft - pijnlijk } \\
& \text { schwatzhaft - babbelachtig } \\
& \text { wehrhaft - weerbaar } \\
& \text { zaghaft - timide, schuchter }
\end{aligned}
$$

Allenfalls für die semantisch motivierte Untergruppe, geneigt sein, das zu tun, was durch das Basisverb ausgedrückt wird' lässt sich eine zumindest partielle Korrespondenz zwischen -haft und -achtig feststellen (vgl. naschbaft und schwatzhaft). ${ }^{17}$ Der semantische Typus schmerzhaft dagegen (eine schmerzhafte Wunde schmerzt) wird im Niederländischen nicht mit -achtig realisiert.

Eine letzte Besonderheit des niederländischen -achtig ist der denominale Typ, dessen Semantik Booij (2002) als, liking X`angegeben hatte. Dieser Typ hat, soweit ich sehe, im Deutschen keine morphologische Entsprechung. Es geht um Ableitungen von Substantiven, wobei das Adjektiv eine - wie

\footnotetext{
16 Snoepachtig kann auch denominal interpretiert werden und würde dann eher zu den Beispielen unter (25) gehören: ik ben niet zo snoepachtig - ich stehe nicht so auf Süßigkeiten.

17 Vgl. aber beispielsweise vergeetachtig - vergesslich (*vergesshaft).
} 
Maesfranckx und Taeldeman (1998) es nennen - ,affektive` Bedeutung hat. Diese Bedeutung würde man im Deutschen vielleicht am ehesten mit dem Ausdruck ,auf etwas stehen' wiedergeben:

Niederländisch: Ik ben niet zo bierachtig/kerkachtig/museumachtig/taartachtig/visachtig ...

Deutsch: Ich stehe nicht so auf Bier/Kirche/Museum/Torte/Fisch ...

Nach dieser Fallstudie zur Adjektivableitung möchte ich im folgenden Abschnitt auf einige Implikationen und Konsequenzen für die kontrastive Sprachwissenschaft und für die morphologische Theorie hinweisen.

\section{Theoretische Implikationen}

Die Fallstudie hat gezeigt, dass Wortbildungsmuster im Laufe der Zeit einem Prozess unterliegen, den Franz Rainer als ,semantic fragmentation' bezeichnet hat. Es bilden sich Untergruppen von semantisch zusammengehörigen Wörtern. Diese semantischen Nischen zeichnen sich durch ein hohes Maß an Kohärenz aus, sowohl im Hinblick auf die möglichen Basiswörter als auch im Hinblick auf die Semantik der resultierenden Derivate. Nicht selten geht es um Unterkategorien, die auch im Hinblick auf Erweiterbarkeit und Produktivität ein Eigenleben führen. So können sich innerhalb einer formalen Wortbildungskategorie Produktivitätsinseln herausbilden, wie beispielsweise die denominalen Adjektive auf -haft und -achtig auf der Basis von Personenbezeichnungen.

Hier bestätigt sich also in gewisser Weise Aronoffs bekanntes Diktum "productivity goes hand in hand with semantic coherence" (Aronoff 1976, S. 45) (auch wenn er das selber wohl etwas anders gemeint hat und eher auf ganze morphologische Kategorien bezogen hat).

Eine zentrale Frage jeder morphologischen Theorie betrifft den Abstraktionsgrad, der für die Beschreibung notwendig ist. Innerhalb der theoretisch orientierten morphologischen Literatur findet man immer wieder die Forderung nach größtmöglicher Generalisierung. Generative Wortbildungsregeln spezifizieren dann typischerweise die Wortart des Basisworts, das Affix, die Wortart des resultierenden Wortes sowie eine sehr abstrakte Charakterisierung der Wortbildungsbedeutung. Monosemie wird als wünschenswert gesehen und es wird versucht von den konkreten Wortbedeutungen zu abstrahieren, um zu einem 1:1-Verhältnis zwischen formalem Prozess und Semantik zu gelangen.

Das extreme Gegenstück zu dieser Position ist die lexikologisch-lexikografische Position, die vor allem von philologisch orientierten Sprachwissenschaftlern des vergangenen Jahrhunderts vertreten wurde. Diese Haltung wird gut durch ein Zitat von Leo Spitzer illustriert: 
Es ist [...] notwendig, jede einzelne Wortbildung zu individualisieren. Der Grundsatz der neueren Lexikographie ,jedes Wort hat seine eigene Geschichte' gilt auch für die Wortbildungslehre. Die Serien von Wörtern gleicher Endung oder Vorsilbe sind ,mirages', sie zerfallen bei mikroskopischer Betrachtung ebenso wie die Lautgesetze: [...] So mündet denn die Wortbildungslehre von selbst in das Wörterbuch ein. (Spitzer, Archivum Romanicum 7, 1923, S. 198 (nach Rainer 2003, S. 197))

Zielführender als diese beiden Maximalpositionen erscheint mir eine Zwischenposition. Die präsentierte Fallstudie ist meines Erachtens ein Indiz für die Richtigkeit einer Lexikon-Konzeption, wie sie u.a. von Ray Jackendoff und insbesondere auch in der Konstruktionsgrammatik vertreten wird. Das Lexikon wird dann nicht mehr als eine Liste von Wörtern und Affixen aufgefasst, ,it is a repository of whatever pieces of linguistic structure have to be stored in long-term memory. Some of these pieces are relatively idiosyncratic, and some include variables that must be filled productively or semiproductively“, schreibt Jackendoff (2008, S. 18).

Wortbildungsmuster können als Konstruktionen aufgefasst werden, als ein Schema mit Variablen, und sind so Teil des Lexikons. Wichtig ist in diesem Zusammenhang die Konzeption des Lexikons als ,hierarchisches Lexikon', wie sie u.a. von Geert Booij (2010) entworfen wird. Wortbildungsmuster können - als Konstruktionen - mit verschiedenen Abstraktionsgraden im Lexikon präsent sein, wobei die verschiedenen Konstruktionen dann durch ,inheritance' (also Vererbung) miteinander und mit den involvierten Wörtern verbunden sind.

Subschemata erlauben Generalisierungen über Subsets von morphologisch komplexen Wörtern innerhalb einer morphologischen Kategorie (Booij 2010, S. 55). Das Subschema definiert dann die spezifischen Eigenschaften dieses Subsets, wie beispielsweise formale oder semantische Besonderheiten der Basiswörter oder eine spezifische Bedeutung der Ableitungen. Somit können auch Unterschiede zwischen Subsets im Hinblick auf ihre Produktivität verantwortet werden, wie Booij anhand einer Untersuchung zu Verben mit dem Präfix ver-im Niederländischen feststellt:

The differences in productivity can be accounted for assuming subschemas for the productive cases, dominated by a general schema for all ver-verbs, that expresses that this prefix always creates verbs. Such subschemas identify the regions of productivity of a word formation process. (Booij 2010, S. 53)

Derartige Phänomene haben wir auch anhand der Fallstudie feststellen können. Es gibt für Adjektive mit -haft und -achtig in beiden Sprachen generelle Wortbildungsmuster, unter denen sich aber deutliche semantische Nischen und Produktivitätsinseln unterscheiden lassen, die man wiederum als - weniger abstraktes - Muster beschreiben kann. Diese erben die Eigenschaften von allgemeineren Mustern und spezifizieren sie im Hinblick auf die formalen und/oder semantischen Besonderheiten und im Hinblick auf die Produktivität. 
Konkret könnte man beispielsweise ein allgemeines, abstraktes Muster (,Schema) für denominale Ableitungen annehmen, zu dem es ein Subschema für Vergleichsbildungen gibt. Im Fall des Niederländischen ist dieses Subschema produktiv: Es lassen sich neue Ableitungen bilden (wie freakachtig oder Google-achtig). Im Fall des Deutschen muss das Subschema für die Vergleichsbildungen enger definiert werden. Es ist nur mit Personenbezeichnungen als Basis produktiv (freakhaft). Ansonsten korrespondieren die niederländischen Vergleichsbildungen auf -achtig nicht mit - baft sondern mit -artig (googleartig).

Die Annahme von Subschemata erlaubt somit die Gegenüberstellung von unterschiedlichen Wortbildungsmustern für semantische und andere Wortbildungsnischen. Zusammengehörige Reihen von Adjektiven mit - haft bzw. -achtig können in der jeweils anderen Sprache auch mit einem Subschema eines anderen Wortbildungsmusters korrespondieren. Das betrifft nicht nur die denominalen Vergleichsbildungen, sondern auch die oben behandelten Farbadjektive (groenacbtig-grünlich) oder die topographischen Adjektive, wo -achtig mit dem deutschen -ig korrespondiert. Es ergibt sich ein komplexes Netzwerk von Schemata und Wörtern, die einander in unterschiedlicher Weise entsprechen, wobei die genauen Entsprechungen häufig die Geschichte der involvierten Wortbildungsmuster reflektieren.

\section{Schlussbemerkungen}

Wortschatz und Wortbildung erscheinen im Sprachvergleich auf den ersten Blick oft unsystematisch oder gar chaotisch und für Generalisierungen weitgehend unzugänglich. Wörter und Wortbildungsmuster haben im Laufe der Geschichte allerlei idiosynkratische Eigenschaften entwickelt, Divergenzen und Unterschiede dominieren das Bild. Dennoch ist die kontrastive Analyse meines Erachtens geeignet, unser Verständnis des Funktionierens von Wortbildung zu verbessern. Durch die Einbeziehung der diachronen Perspektive sind wir in der Lage, die Faktoren und Prozesse zu identifizieren, die zu Divergenz und Konvergenz führen. Und die genaue kontrastive Analyse macht es möglich, Wortbildungsnischen und ,Produktivitätsinseln ${ }^{6} \mathrm{zu}$ identifizieren, die sowohl einzelsprachlich als auch für den Sprachvergleich relevant sind.

Durch die Analyse von Korrespondenzen zwischen Wortbildungsmustern (semantisch, formal, im Hinblick auf Produktivität) stößt man auf die relevanten semantischen Nischen, auf Reihen von Wörtern, die durch Analogie zusammengehalten werden und erweitert werden können. Die Tatsache, dass diese Nischen sich dann im Niederländischen und im Deutschen auch in formaler Hinsicht als kohärente Sets erweisen, spricht meines Erachtens für die präsentierten Annahmen zur Theorie des Lexikons und der 
Wortbildung. Ein hierarchisch strukturiertes Lexikon enthält nicht nur die Wörter sondern auch Wortbildungsmuster bzw. Schemata in unterschiedlichen Abstraktionsgraden. Es erlaubt dadurch die Feststellung von Korrespondenzen (und Unterschieden) zwischen Sprachen auf verschiedenen Niveaus.

Ganz im Sinne der kontrastiven Linguistik lassen sich diese Einsichten meines Erachtens auch für den Fremdsprachenerwerb nutzen. In den mir bekannten kontrastiven Grammatiken wird die Wortbildung in der Regel nur knapp (oder gar nicht) behandelt. Das ist auch nicht weiter verwunderlich, denn wenn man versucht, Affixe oder morphologische Kategorien einander gegenüberzustellen (also beispielsweise, die Adjektive auf -haft' und ,die Adjektive auf -acbtig), so führt das zu völlig unbrauchbaren Resultaten. Erst die genaue Analyse und die Identifizierung relevanter Nischen und Subschemata innerhalb dieser großen Kategorien führt zu sinnvollen Generalisierungen und Gegenüberstellungen, die - eine entsprechende Didaktisierung vorausgesetzt - auch für den Fremdsprachenunterricht fruchtbar gemacht werden können.

\section{Literatur}

Aronoff, Mark (1976): Word formation in generative grammar. (= Linguistic Inquiry Monographs 1). Cambridge/London.

Booij, Geert (2002): The morphology of Dutch. Oxford.

Booij, Geert (2010): Construction morphology. Oxford.

Carr, Charles T. (1939): Nominal compounds in Germanic. (= St. Andrews University Publications 41). London.

Deutscher, Guy (2010): Through the language glass: Why the world looks different in other languages. New York.

Eichinger, Ludwig M. (2000): Deutsche Wortbildung: Eine Einführung. Tübingen.

Fleischer, Wolfgang/Barz, Irmhild (1992): Wortbildung der deutschen Gegenwartssprache. Unter Mitarbeit von Marianne Schröder. Tübingen.

Grimm, Jacob/Grimm, Wilhelm (1854-1960): Deutsches Wörterbuch. 16 Bde. (32 Teilbde.). Leipzig.

Haeseryn, Walter et al. (1997): Algemene Nederlandse Spraakkunst. Tweede, geheel herziene druk. Groningen/Deurne.

Haspelmath, Martin (2001): The European linguistic area: Standard average European. In: Haspelmath, Martin et al. (Hg.): Language typology and language universals. (= Handbücher zur Sprach- und Kommunikationswissenschaft 20). Berlin/New York, S. 1492-1510.

Heine, Bernd/Kuteva, Tania (2006): The changing languages of Europe. Oxford. 
Henzen, Walter (1965): Deutsche Wortbildung. 3., durchges. u. erg. Aufl. (= Sammlung kurzer Grammatiken germanischer Dialekte B-5). Tübingen.

Hüning, Matthias (1997): Het tegaan van een morfologische categorie: over het Middelnederlandse verbaalprefix te-. In: van Santen, Ariane/van der Wal, Marijke (Hg.): Taal in tijd en ruimte. (= SNL-reeks 4). Leiden, S. 23-35.

Hüning, Matthias (2010): Adjective + Noun constructions between syntax and word formation in Dutch and German. In: Onysko, Alexander/Michel, Sascha (Hg.): Cognitive perspectives on word formation. (= Trends in Linguistics. Studies and Monographs 221). Berlin/New York, S. 195-215.

Hüning, Matthias/Schlücker, Barbara (2010): Konvergenz und Divergenz in der Wortbildung. Komposition im Niederländischen und im Deutschen. In: Dammel, Antje/ Kürschner, Sebastian/Nübling, Damaris (Hg.): Kontrastive Germanistische Linguistik. (= Germanistische Linguistik 206/209). Hildesheim/Zürich/New York, S. $783-825$.

Jackendoff, Ray (2008): Construction after construction and its theoretical challenges. In: Language 84, 1, S. 8-28.

König, Ekkehard/Haspelmath, Martin (1999): Der europäische Sprachbund. In: Reiter, Norbert (Hg.): Eurolinguistik - ein Schritt in die Zukunft. Beiträge zum Symposion vom 24. bis 27. März 1997 im Jagdschloss Glienicke (bei Berlin). Wiesbaden, S. $112-127$.

Koziol, Herbert (1972): Handbuch der englischen Wortbildungslehre. 2., neubearb. Aufl. Heidelberg.

Krzeszowski, Tomasz P (1990): Contrasting languages: The scope of contrastive linguistics. (= Trends in Linguistics. Studies and Monographs 51). Berlin/New York.

Maesfranckx, Patricia/Taeldeman, Johan (1998): Polyseem, polyvalent en vaag -achtig. In: Hoekstra, Eric/Smits, Caroline (Hg.): Morfologiedagen 1996. (= Cahiers van het Meertens Instituut 10). Amsterdam, S. 84-105.

Marchand, Hans (1969): The categories and types of present-day English word-formation: A synchronic-diachronic approach. 2., kompl. überarb. u. erw. Aufl. München.

Paul, Hermann (1920): Deutsche Grammatik. Bd. V: Wortbildungslehre. Halle a.S.

Pijnenburg, Willy J. (1993): Honderd jaar -acbtig: Over de wording van een samengesteld suffix. In: Amsterdamer Beiträge zur älteren Germanistik 37, S. 33-48.

Plank, Frans (1981): Morphologische (Ir-)Regularitäten. Aspekte der Wortstrukturtheorie. (= Studien zur deutschen Grammatik 13). Tübingen.

Pümpel-Mader, Maria et al. (1992): Deutsche Wortbildung. Typen und Tendenzen in der Gegenwartssprache. Fünfter Hauptteil: Adjektivkomposita und Partizipialbildungen (Komposita und kompositionsähnliche Strukturen 2). (= Sprache der Gegenwart 80). Berlin/New York.

Rainer, Franz (2003): Semantic fragmentation in word-formation: The case of Spanish -azo. In: Singh, Rajendra/Starosta, Stanley (Hg.): Explorations in Seamless Morphology. New Delhi/Thousand Oaks/London, S. 197-211. 
Tellenbach, Elke (1976): Neuhochdeutsche und neuniederländische Bildungen mit dem Präfix ver-. In: Beiträge zur Geschichte der Deutschen Sprache und Literatur 96, S. 5-63.

van den Toorn, Maarten C. (1984): Van godevolen tot computergestuurd. In: Spektator 13, 6, S. 405-416.

van der Horst, Joop/van der Horst, Kees (1999): Geschiedenis van het Nederlands in de twintigste eeuw. Den Haag/Antwerpen.

van der Sijs, Nicoline (1996): Leenwoordenboek: De invloed van andere talen op het Nederlands. Den Haag/Antwerpen.

van Marle, Jaap (1978): Veranderingen in woordstructuur. In: Koefoed, Geert/van Marle, Jaap (Hg.): Aspecten van taalverandering. Groningen, S. 127-176.

Wandruszka, Mario (1998): Die europäische Sprachengemeinschaft. 2., durchges. Aufl. Tübingen.

Wilmanns, Wilhelm (1899): Deutsche Grammatik. Gotisch, Alt-, Mittel- und Neuhochdeutsch. Zweite Abteilung: Wortbildung. 2. Aufl. Straßburg.

Wilss, Wolfram (1986): Wortbildungstendenzen in der deutschen Gegenwartssprache: Theoretische Grundlagen - Beschreibung - Anwendung. (= Tübinger Beiträge zur Linguistik 304). Tübingen. 